\title{
Do-It-Yourself (DIY) Artificial Pancreas Systems for Type 1 Diabetes: Perspectives of Two Adult Users, Parent of a User and Healthcare Professionals
}

\author{
Syed Haris Ahmed (D) - David L. Ewins · Jane Bridges · Alison Timmis • Nicola Payne \\ Cormac Mooney · Claire MacGregor
}

Received: June 10, 2020 / Published online: July 21, 2020

(C) The Author(s) 2020

\begin{abstract}
The artificial pancreas system or an automated insulin dosing system has been the 'holy grail' for patients with type 1 diabetes and their caregivers who have over the years wanted to 'close the loop' between monitoring of glucose and delivery of insulin. The launch of the Medtronic MiniMed 670G system in 2017 and the subsequent release of the Tandem t:slim with Control-IQ system, the DANA RS pump compatible-CamAPS FX app and the more recent announcement of the Medtronic MiniMed 780G system have come as answers to their prayers. However, in the time taken to develop
\end{abstract}

Digital Features To view digital features for this article go to https://doi.org/10.6084/m9.figshare.12555278.

S. H. Ahmed $(\bowtie) \cdot$ D. L. Ewins · J. Bridges Department of Endocrinology and Metabolic Medicine, Countess of Chester Hospital NHS Foundation Trust, Chester, UK

e-mail: asyedharis76@gmail.com

S. H. Ahmed

School of Medicine, University of Liverpool, Liverpool, UK

A. Timmis

Department of Children's and Adolescent Services, Countess of Chester Hospital NHS Foundation

Trust, Chester, UK

N. Payne · C. Mooney · C. MacGregor

Countess of Chester Hospital NHS Foundation

Trust, Chester, UK and launch these commercial systems, creative and ebullient parents of young patients with type 1 diabetes, along with other patients, technologists and healthcare professionals have developed mathematical models as software solutions to determine insulin delivery that in conjunction with compatible hardware have helped 'close the loop'. Under an umbrella movement \#WeAreNotWaiting, they have, as a community, refined and disseminated technologies that are open source and ubiquitously available as do-it-yourself (DIY) closed-loop systems or DIY artificial pancreas systems (APS). There are presently three systems-OpenAPS, AndroidAPS and Loop. We present perspectives of two patients, parent of a patient, and their healthcare providers; the users spanning an age spectrum most likely to use this technology-a child, an adolescent in transitional care and a 31-yr old adult patient, highlighting how looping has helped them self-manage diabetes within the routine of their lives and the challenges they faced.

Keywords: Artificial pancreas systems; Type 1 diabetes; Continuous glucose monitoring; Doit-yourself (DIY); Insulin pump therapy; Hypoglycaemia; Time-in-range 


\section{Key Summary Points}

In this commentary, two adult patients and the caregiver of a patient, with type 1 diabetes share their experiences on DIY artificial pancreas systems. Their healthcare providers provide clinical perspectives.

The users' quality of life and ability to selfmange diabetes have been enhanced by this open source technology. By adopting DIY systems, they have joined a \#WeAreNotWaiting community that is willing to innovate, experiment and promote technology, modelled on the function of a healthy human pancreas.

Further research would help UK regulatory bodies develop the guidance, for healthcare professionals, to support DIY APS users.

\section{PERSPECTIVES OF TWO ADULT USERS AND PARENT OF A USER}

\section{Nicola's Story}

I am a 31-year veterinary surgeon living with type 1 diabetes since 2008. With a scientific background I have always strived for the "perfect" control of my condition, but this has always come with a huge and unrelenting mental daily burden. Around 18 months ago I stumbled across the concept of looping online and began to learn about this automated insulin delivery system. I was excited at the possibility of having some of my disease burden alleviated. Loop utilises inputted data and glucose readings to predict future blood glucose level and will adjust basal rates up or down accordingly. The algorithm alters this decision every $5 \mathrm{~min}$ and the predicted trend of your blood glucose is shown, alongside active insulin, insulin delivery and active carbohydrates. This

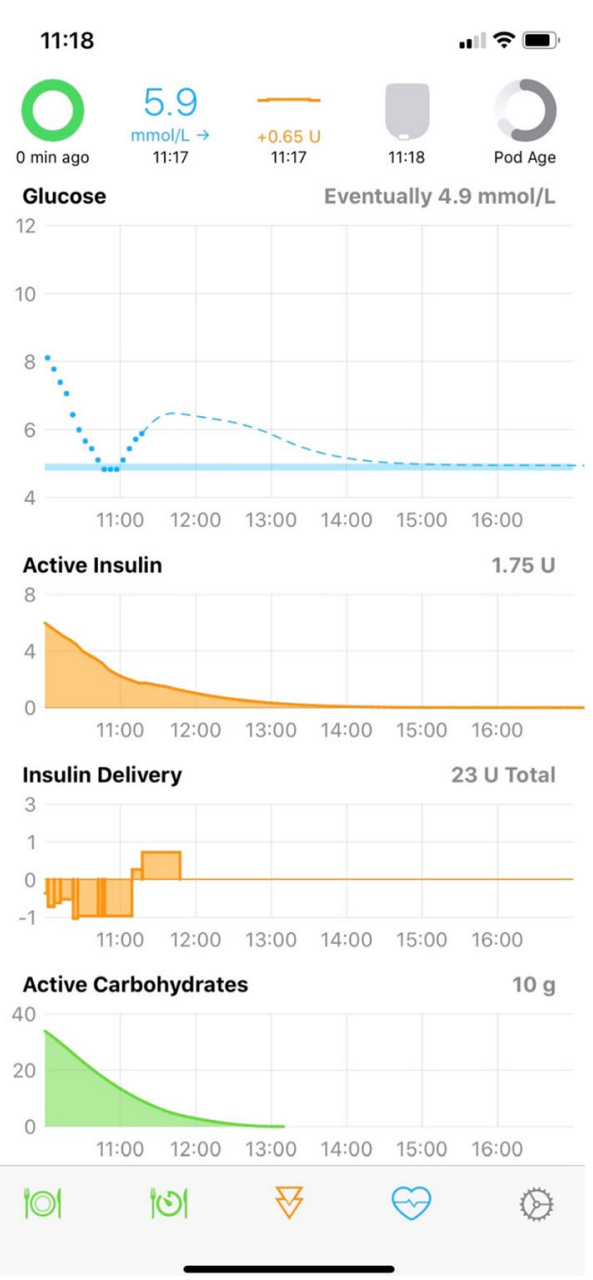

Fig. 1 Loop app home screen on an iPhone showing active carbohydrates, insulin delivery, active insulin

information is presented on the Loop home screen (Fig. 1). It is recommended to set duration of insulin action (DIA) to $6 \mathrm{~h}$ with Loop, longer than that recommended for traditional pumping to prevent insulin stacking.

During the transition to Loop one of the challenges was learning to understand and trust the different recommendations given for boluses which did not always correlate with what I would have calculated using traditional pump therapy. It took time to get used to the concept that bolus and basal are more interchangeable with Loop, so an increased basal after a meal needs to be considered as an extended bolus would on traditional pump therapy. Switching from using a Personal Diabetes Manager (PDM) to my phone and using my Apple watch 
allowed me to more discretely manage my condition but this came with the practical challenge of ensuring I always had enough phone battery power or a charger with me. I also had to ensure I always had my RileyLink with me, but soon got used to this. At home it can be in another room and still communicate effectively but I try to keep it with me as much as possible.

I set up a Nightscout profile which collates all of my continuous glucose monitor (CGM), insulin delivery and carbohydrate information to produce reports which can then be viewed by healthcare professionals.

Loop utilises "carbohydrate absorption time" rather than extended boluses. Carbohydrate absorption is tracked over time and basal rates are adjusted accordingly after the initial upfront bolus. A progress bar shows carbohydrate absorption, helping the user to learn different foods' absorption profiles so more educated guesses can be made in future for the same meal, minimising the risk of hypoglycaemia. High fat meals such as pizza are handled better with Loop than I managed with conventional pumping and whilst I cannot say that Loop stops any blood glucose rises, it has reduced my time out of range and the height of any spikes, reducing the requirement for repeated correction doses. I still find that pre-bolusing is the most successful way of preventing glucose spikes after meals; there is also a user preset "pre-meal" setting which can be enacted for up to $1 \mathrm{~h}$ before eating, allowing more insulin on board when the meal is digested.

Exercise-induced hypos are less common for me on Loop, as the algorithm anticipates any impending low blood glucose and reduces the basal rate. Carbohydrate portions for correction of hypoglycaemia are lower, for example I now require 1-2 dextrose tablets instead of 3 to promptly correct hypoglycaemia and avoid post-correction hyperglycaemic excursions. Overrides and temporary targets are a valuable feature to deal with scenarios where insulin requirements differ from baseline. I use these settings commonly for work, exercise and post exercise to combat hypoglycaemia (Fig. 2).

There are some downsides to the Loop system; although for me, these are far outweighed

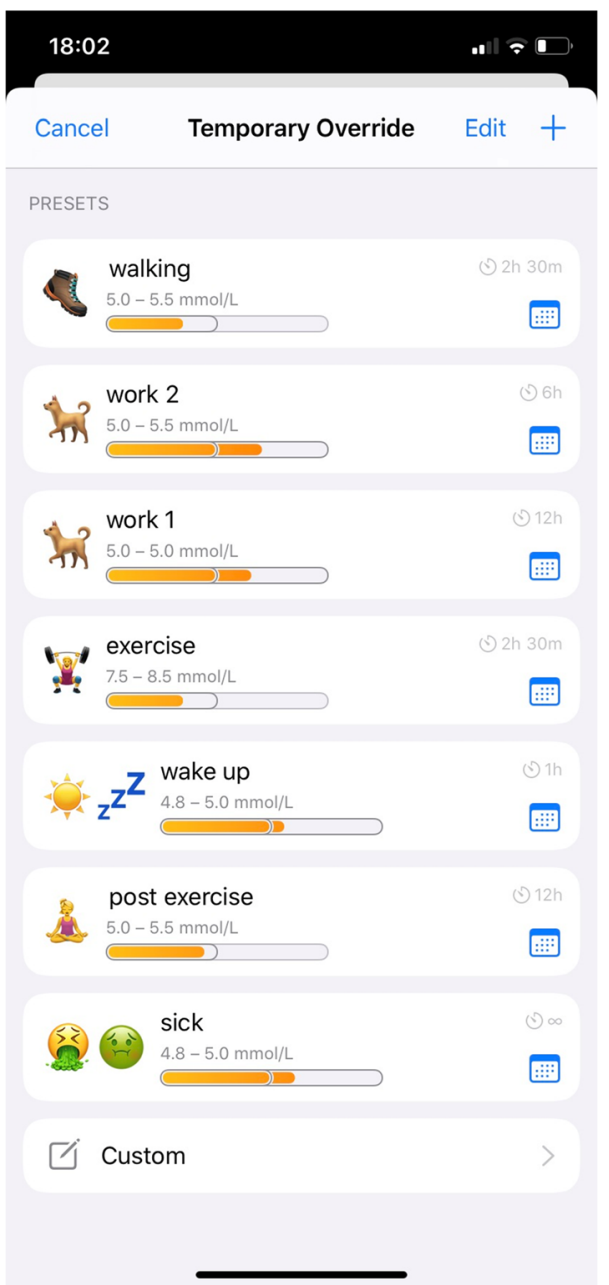

Fig. 2 Temporary preset overrides for different situations

by the positives. It requires a significant ongoing financial input and a substantial time to read and understand the algorithm and to set up the system. Being somewhat technologically savvy is also a bonus, although the instructions for setting up the system are comprehensive and there is a community of people available online to help. However, new users should be under no illusion that you simply "plug in and go"-Loop needs accurate settings such as carbohydrate ratios, insulin sensitivity and target glucose ranges to work safely and effectively. More practical pitfalls with looping relate to the CGM used; I use the Dexcom G6 which I have found to be very reliable and accurate in its readings; however, on the infrequent occasion that there is a sensor error, the lack of CGM data 
means that Loop cannot function. Insulin delivery will continue as the OmniPod will revert back to its preset basal rate but if there are any carbohydrates on board then Loop cannot make its basal adjustments to compensate for this.

After just under 12 months of looping, I found that my initial monetary, effort and time investment paid off as improvement in my overall control and quality of life.

My HbA1c has reduced from $6.5 \%$ to $5.5 \%$ and my time-in-range (TIR) is $76 \%$ for my personalized target range of $3.9-7.8 \mathrm{mmol} / \mathrm{l}$ (90-95\% for the standard target range of 3.9-$10 \mathrm{mmol} / \mathrm{l}$ ) with less post-meal hyperglycaemia (Fig. 3). Improvements in my quality of life include an undisturbed overnight sleep, less time spent making decisions regarding insulin management and improved confidence when exercising.

Loop technology is undergoing constant development, with input and suggestions from users, researchers and healthcare specialists. I am excited to see where this project goes next and cannot express my gratitude to those involved in its creation/maintenance and to my healthcare team for supporting me in my decision to use a combination of licensed and unlicensed products in an off-label manner.

\section{Cormac's Story}

I was diagnosed with type 1 diabetes in January 2018, aged 16 and began treatment with a multiple injection regimen (MDI) and glucose monitoring with an Accu-Chek meter. After approximately 8 months of maintaining good control and an HbA1c of below $50 \mathrm{mmol} / \mathrm{mol}$, I began to find it more difficult to keep within a healthy range. I left one clinic appointment in tears, feeling like I'd failed with an HbA1c result of above 70. Some days I didn't want to see the blood glucose levels so I didn't carry out many finger prick tests; other days I checked obsessively with over 20 checks being recorded on my meter. These feelings affected my dietary habits and, on some days, I refused to eat as I felt eating wasn't worth the bother of doing the check or the injection. After sharing these thoughts with my diabetes specialist nurse (DSN), I was offered the FreeStyle Libre. This felt like a positive step in my management as it would alleviate some of my finger prick checks. However, decisions to scan, when to scan and then to decide on the correct bolus were everpresent. It was also frustrating not knowing what was happening between checks and not knowing what was going to happen to my glucose levels in the near future.

I have a general interest in technology and my research led me to an article which described the MiaoMiao, a device that was capable of transforming the FreeStyle Libre into a CGM. I was fortunate that my parents agreed to purchase a MiaoMiao for me in May 2019 which, for the first time, allowed constant monitoring of glucose levels. In addition, using Nightscout, I was able to see predicted glucose levels and share my data with my parents and my DSN. My parents are conscientious supporters in my management of diabetes and sharing the data with them meant that our conversations
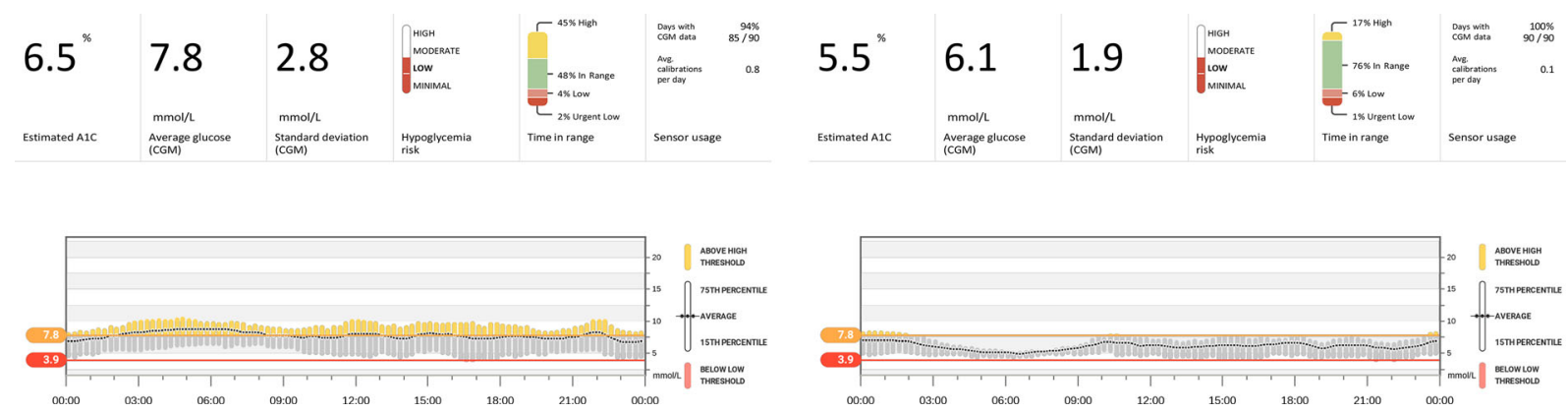

Fig. 3 A 90-day average before (left) and after (right) Nicola started using looping shows improvements in time-in-range (TIR) from $48 \%$ to $76 \%$, in the average glucose and standard deviation from $7.8 \pm 2.8$ to $6.1 \pm 1.9 \mathrm{mmol} / \mathrm{l}$ 
weren't solely about blood glucose levels anymore! Nightscout also predicts HbA1c values, meaning that I could attend clinic appointments with some idea of how the conversation with the consultant was going to go.

My research also led me to discover DIY APS (looping) with the potential to connect my FreeStyle Libre/MiaoMiao combination to a suitable insulin pump via a device called a RileyLink. I was supported in my decision to move from MDI to the OmniPod in June 2019. I chose the OmniPod for its compatibility with the DIY APS system. I spoke at length to my DSN about my intention to try DIY APS; she was supportive of this patient-led management of my diabetes, whilst also ensuring that I was aware that looping is not currently regulated. After I had used the OmniPod for approximately 4 weeks with its PDM, my parents agreed to purchase a RileyLink in July 2019. Whilst waiting for its delivery from USA I thoroughly read through Loop docs online, joined looping, AndroidAPS and OpenAPS groups on social media and began to build the iOS Loop app. I found the creation of the app fairly straightforward, following the instructions in the Loop docs. The connection between the Riley Link and the OmniPod was almost identical to the process of connecting the OmniPod to the PDM. I initially used the Loop app in its open loop mode which requires user verification and confirmation of all decisions made by the app. I found that all the suggested decisions were sensible, much more frequently than I would have made independently (every $5 \mathrm{~min}$ ) and put a greater weighting on the predicted glucose levels than I would have previously done. Figure 4 shows how I loop with the different components.

I felt confident in the open loop and the decisions that were being suggested, so, on the second evening, I decided to close the loop to see what would happen. It was not uncommon for me to experience nocturnal hypos, but overnight the glucose levels remained stable. Since then I have been using the closed-loop configuration with consistently good results. I have seen my HbA1c level drop to $42 \mathrm{mmol} / \mathrm{mol}$ with a reduction in the number of hypo and hyper episodes and around $80 \%$ of my time in range (Fig. 5).

In November 2019 I attended a looping conference in London where members of the looping community shared advice and

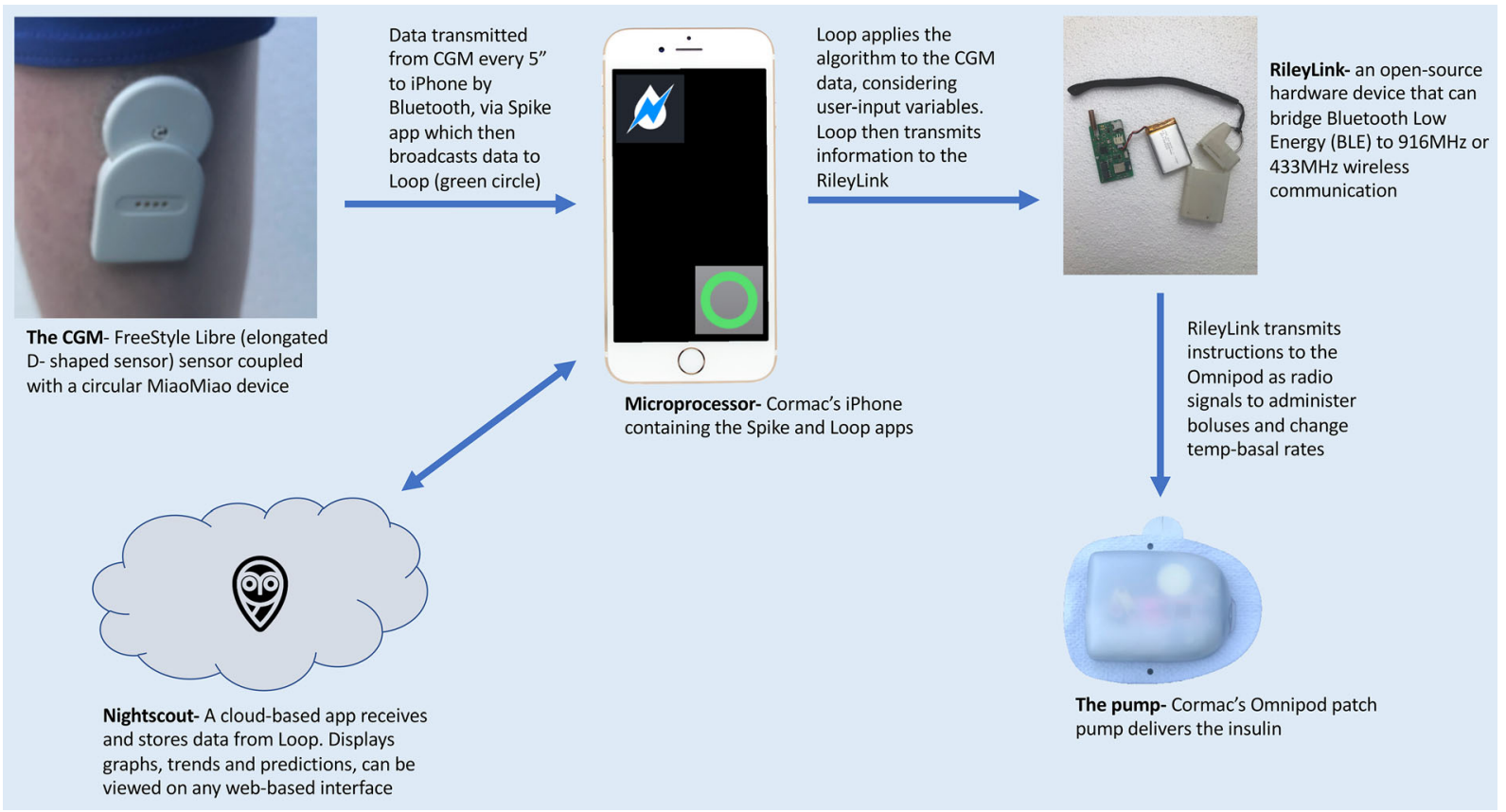

Fig. 4 Diagram describing how different components of Cormac's APS communicate to close the loop 


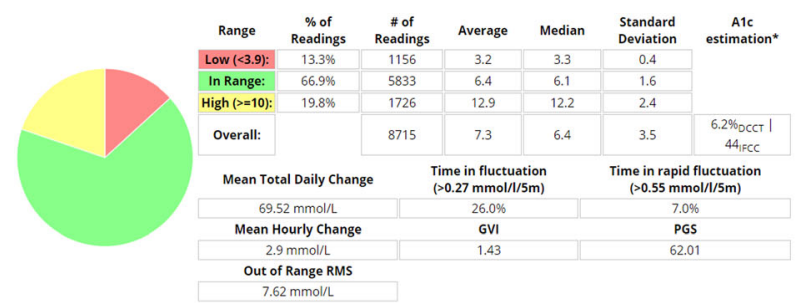

Fig. 5 Cormac's 30-day glucose distribution data pre (left) and post (right) looping shows reduction in hypoglycaemia from $13.3 \%$ to $6.7 \%$, increase in time-in-range (TIR) from

experiences. I was advised to use the Loop with caution; if in doubt, to open the Loop and take back control. I have not experienced any software issues since looping but do experience occasional highs which are corrected by the Loop without subsequent overcorrection lows. My hypos are now less frequent and less severe. Looping has had a positive effect on my mental health. I am grateful that my medical team understand and support my decision to undertake this method of diabetes management. I now perceive diabetes to be less of a burden and less time consuming in my day to day life. The impact of having diabetes on my academic career is reduced as I now spend less time extracting myself from lessons and I am confident that my Loop is automating insulin delivery correctly.

\section{Harry's Story}

My name is Claire, and my son Harry was diagnosed with type 1 diabetes in April 2013 just 2 weeks after his third birthday. Harry used MDI as a way of treatment until December 2015 when he switched to the Animas Vibe pump.

In November 2015 we were given the opportunity to trial the Dexcom G4 CGM. This was a game changer for us. It was as though the mask I had been wearing since Harry's diagnosis had finally been lifted. I suddenly felt able to care for my child in a way I never thought I could. From this day forward, I made it my mission to understand and try to keep up with all the latest diabetes technology available. I needed my boy to live his best life.

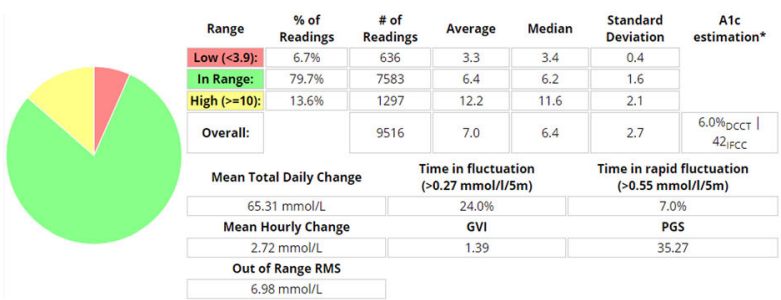

$66.9 \%$ to $79.7 \%$, improvement in glycaemic variability index (GVI) from 1.43 to 1.39 and in patient glycaemic status (PGS) from 62.01 to 35.27

I joined some new Facebook groups, which was the start of our next exciting diabetic journey. Reading what other parents do is a great way of learning new tricks and finding out about new technology that is available. I came across the word "Nightscout" over and over so decided to find out what exactly this was and how I could have it!

Nightscout, or CGM in the cloud, was developed by parents of children with type 1 diabetes and is an open-source DIY project that allows real-time access to CGM data via your own personal website and it completely changed our lives. It gave us freedom that we simply hadn't had since before diagnosis, freedom to sleep easier, to relax when my boy was in another room or playing in the garden, to allow him to be a child and not have to be monitored so closely anymore; it gave me my first taste of remote monitoring, and I loved it!

Fast forward to late 2018, a close friend of mine (someone I met online whilst setting up Nightscout) had set up, and been using flawlessly, a system called Android APS with her daughter. Something I knew very little about, a system I believed would be too difficult for me to set up, so I put off reading too much about its brilliance, as to not get my hopes up. However, as always curiosity got the better of me, and I asked for help. I couldn't let my fear of failure stop me trying.

February 2019, during the half-term school break, I finally set up my very own DIY APS for Harry. Over a year later and I am shocked how amazing and clever this system is.

Currently the system we use includes Dexcom G6 CGM and an Accu-Chek Spirit Combo insulin pump, which Android APS controls via 


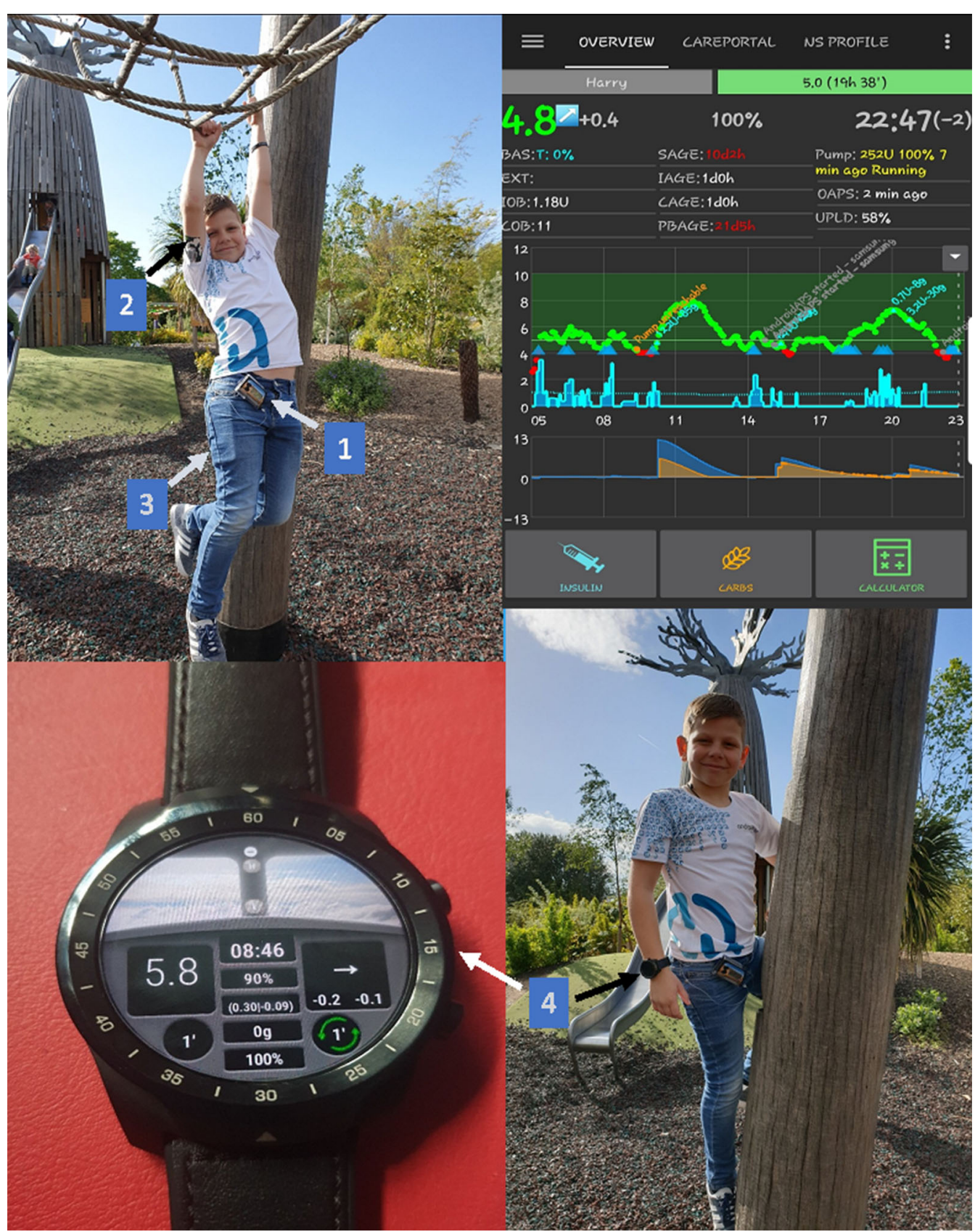

Fig. 6 Top left, bottom left and right-Harry having fun camping. Top right-Harry's AndroidAPS home screen; components of Harry's APS system: 1, Accu-Chek Spirit

Bluetooth (Fig. 6). To see it in action still has me amazed.

Originally, we set up using a Samsung J4+ which worked brilliantly until we switched from Dexcom G5 to G6 in December 2019, when it was then no longer compatible, so we
Combo Insulin pump; 2, Dexcom G6; 3, Android smartphone Xiaomi Mi A2 lite; 4, TicWatch Pro Smartwatch

purchased the Xiaomi Mi A2 Lite which has been working flawlessly since. Harry also wears a TicWatch Pro smartwatch, which he can also bolus by entering the carbs and it will calculate the insulin needed. 
Harry is 10 years old now and becoming more independent so this is really what pushed me to get this set up, as I'm sure before long he will want to take the reins off me completely and I wanted him to be fully prepared and have the best system to work with, and you can't get much better than this.

Before APS was in our lives, I struggled to sleep. I micromanaged Harry's diabetes to the best of my ability 24/7 and was quite hard on myself when things didn't quite go to plan; and let's face it, diabetes often doesn't play fair! Harry's HbA1c has never been of concern and has always been in range. This was never my incentive; bringing back some normality into our lives is what I craved, what we dreamed of. Android APS has allowed me to take a step back; it has allowed me to take a deep breath and see that my son can survive, and survive well, without my constant and often annoying, interference.

The biggest change for me though is Harry's confidence. He says he feels more like his friends now; he is happier, as though it has taken away some of the burden for him. In school he rarely has to give diabetes a second thought anymore. He knows the system he has is constantly working at keeping him safe and he has learned to trust it. He understands how to respond to the alarms, and knows that if he does this, he feels better and so there are less interruptions on his day. He isn't able to give himself correction insulin yet, without supervision, but he knows I am always keeping an eye on his glucose readings from wherever I am, and he can see when I send a text to give him a bolus so he can relax again knowing that things are under control. The remote bolusing via text message is brilliant; it means I never have to interrupt his learning or, more importantly, his play time, just to give insulin. Which when you're 10 is kind of a big deal.

Even though APS is taking the lead, I still feel completely in control; I am the one calling the shots. I can also turn it off via a press of a button, which I have only done when encountering faulty sensors, but it's good to know the option is there should you need it. APS isn't a quick fix; we still get highs and lows, but just not as severe or as often as we did without it.
Our hypo treatment has halved since we have been looping which speaks volumes in itself, whilst still keeping an HbA1c anyone should be proud of. Diabetes is tough, but having an APS to help makes life that little bit easier to manage.

\section{PHYSICIAN PERSPECTIVE}

\section{The Artificial Pancreas System (APS)}

An APS is a system consisting of an insulin pump, a CGM device and a central microprocessor integrating the data to provide automated insulin delivery (AID) based upon interstitial fluid glucose readings and other variables. It is a closed-loop system hybridised to integrate manual data such as carbohydrate intake, glucose targets and other profiles.

A DIY APS or DIY hybrid closed-loop system (known as looping) is created by the user using source code freely available from software development platforms such as GitHub. There are currently three main systems in DIY looping: OpenAPS, Android APS and Loop (Table 1). The current three commercially available APS systems: the Medtronic MiniMed 670G, the Tandem t:slim with Control-IQ and the DANA RS pump compatible-CamAPS FX app, lack the customization and flexibility afforded by DIY loop systems. The MiniMed 780G system secured the CE marking in June 2020 and is yet to be commercially launched; compared to the 670G, it has added features, prominent of which are, the ability to adjust target setting to as low as $5.5 \mathrm{mmol} / \mathrm{L}$ and Bluetooth connectivity with compatible iOS and Android systems.

DIY looping was driven by the desire of people living with diabetes (\#WeAreNotWaiting) to devise a system mimicking the human pancreas to reduce the burden of daily diabetes management. The idea that an algorithm could be designed to predict glucose trends and shared via the cloud was conceived by John Costik in 2013 and developed as the Nightscout project [1]. Dana Lewis, after successfully trialing this concept with her CGM, worked with John Costik to further refine the algorithm. Later they worked with Ben West and in 2014 
Table 1 The three DIY APS systems and associated technology

\begin{tabular}{|c|c|c|c|}
\hline Systems & OpenAPS & AndroidAPS & Loop \\
\hline Designers & $\begin{array}{l}\text { Dana Lewis, Scott Leibrand, } \\
\text { Ben West }\end{array}$ & Milos Kozak, Adrian Tappe & $\begin{array}{l}\text { Nate Racklyeft, Peter } \\
\text { Schwamb }\end{array}$ \\
\hline Pumps & $\begin{array}{l}\text { Old Medtronic (versions } \\
\text { before 2011) }\end{array}$ & $\begin{array}{l}\text { Dana R, Dana RS } \\
\text { Roche Accu-Chek Combo } \\
\text { Roche Accu-Chek Insight }\end{array}$ & $\begin{array}{l}\text { Old Medtronic } \\
\text { OmniPod Eros pods }\end{array}$ \\
\hline CGM systems & $\begin{array}{l}\text { Dexcom G4, G5, G6, } \\
\text { Medtronic Enlite or MiniMed } \\
\text { Paradigm REAL-time Revel, xDrip+ } \\
\text { FreeStyle Libre with MiaoMiao } \\
\text { Synchronizes with Nightscout }\end{array}$ & $\begin{array}{l}\text { Dexcom G4, G5, G6, } \\
\text { Eversense, Medtronic } \\
\text { Guardian or Enlite, xDrip+ } \\
\text { FreeStyle Libre with MiaoMiao } \\
\text { Can synchronize with } \\
\text { Nightscout }\end{array}$ & $\begin{array}{l}\text { Dexcom G5 or G6 (or } \\
\text { G4 with Share) } \\
\text { FreeStyle Libre with } \\
\text { MiaoMiao } \\
\text { Can synchronize with } \\
\text { Nightscout }\end{array}$ \\
\hline $\begin{array}{l}\text { Computing hardware } \\
\text { (rig), bridging } \\
\text { device }\end{array}$ & $\begin{array}{l}\text { Small computer (e.g. Intel } \\
\text { Edison or Raspberry Pi) and a } \\
\text { radioboard/stick } \\
\text { (Explorer Board for Edison } \\
\text { or Explorer HAT for Pi) } \\
\text { xDrip Wireless Bridge } \\
\text { (Wixel) for xDrip users }\end{array}$ & $\begin{array}{l}\text { Android phone, xDrip Wireless } \\
\text { Bridge (Wixel) for xDrip } \\
\text { users }\end{array}$ & $\begin{array}{l}\text { iPhone, RileyLink } \\
-916 \mathrm{MHz} \text { for } \\
\text { Medtronic } \\
-433 \mathrm{MHz} \text { for } \\
\text { OmniPod Eros }\end{array}$ \\
\hline User interface devices & $\begin{array}{l}\text { Pebble smart watch } \\
\text { Android phone } \\
\text { iPhone } \\
\text { PC running Windows or } \\
\text { Mac }\end{array}$ & $\begin{array}{l}\text { Android phone } \\
\text { Smart watch }\end{array}$ & $\begin{array}{l}\text { iPhone } \\
\text { Apple watch }\end{array}$ \\
\hline Operating system & & Android & iOS \\
\hline Algorithm & OpenAPS & OpenAPS & Loop \\
\hline $\begin{array}{l}\text { Communication } \\
\text { with } \\
\text { rig }\end{array}$ & Radiofrequency & Bluetooth & Bluetooth \\
\hline
\end{tabular}

devised a system that allowed them to control a pump in conjunction with the CGM and the predictive algorithm to 'close the loop'launching the OpenAPS movement [1].

Their prototype DIY rig consisted of a microprocessor holding the algorithm, a radio stick for communication, a pump and CGM. In Table 1, we have detailed the different components and software interfaces required for the current three types of systems.

The first algorithm released, orefo (OpenAPS Reference Design Zero), has evolved over time with features to limit hyperglycaemia whilst minimising hypoglycaemia. The newer oref1 algorithm instructs the pump to give small 'supermicroboluses' in response to post-prandial rises in the blood glucose. 
Table 2 Pros and cons of DIY APS as compared to traditional sensor-augmented pump therapy

Pros Cons
Cons

Reduction in number and severity of hypoglycaemic episodes
Improved glycaemic stability, reduced variability

Improvement of time-in-range (TIR)

Reduced cognitive burden, as less time spent thinking and planning about diabetes. Less time spent on diabetesrelated activity, giving more time and cognitive space to focus on other activities in life

Greater piece of mind and psychological reassurance to users (and caregivers) regarding variables such as overnight hypoglycaemia etc., leading to a better quality of sleep

Improved overall quality of life

More responsive to an individual's change in physiology

Remote monitoring option by cloud-based system such as Nightscout

Supportive online DIY community

Regular software updates and refinement of algorithms that are user-sensitive, and assist users to manage glycaemia with minimal user input

Better flexibility and customization allowing communication with portable devices e.g. Harry's uses his smartwatch to bolus from his pump
Limited interoperability which means limited number of pumps and CGM devices compatible for DIY systems. However, the pool of different models that can 'loop' is gradually expanding

Financial costs for additional hardware not covered by insurance

Warranty will not cover accidental damage of the pump or CGM, caused by use within an unlicensed system

Additional hardware components such as the RileyLink/ xDrip wireless bridge/radioboard/stick/minicomputer along with the battery and cables will have to carried along in a separate case/purse or pocket, for the system to work. However, the newer hybrid closed-loop systems have Bluetooth connectivity, obviating the requirement of intermediate hardware

Perceived and real technical barriers limiting individual patient uptake

Perceived and real lack of knowledge and understanding of DIY systems among healthcare professionals

Requires investment of time and effort to learn and set up the system

Faster drain of pump and smartphone batteries

Lack of randomised clinical trials to assess safety and efficacy

Neither medically authorised nor regulated

Currently OpenAPS and AndroidAPS implement the orefo algorithm, whereas Loop uses a different algorithm wherein insulin is adjusted on the basis of future predicted glucose levels. Cormac's APS in Fig. 4 exemplifies how the different components of DIY system work in tandem as an automated loop. Cormac uses a FreeStyle Libre with MiaoMiao whereas Nicola and Harry use a Dexcom G6 for CGM; Cormac and Nicola use the Spike and Loop apps on their iPhones, have RileyLinks as intermediate hardware, and use an OmniPod patch pump for insulin delivery; whereas Harry uses an Android-based Xiaomi Mi A2 Lite phone as the microprocessor which 
communicates directly with his Accu-Chek Spirit Combo insulin pump by Bluetooth. One can see the importance of 'interoperability' of the different devices in allowing patients to make an informed and tested choice on the right components that would work well for them.

\section{What Is the Supporting Evidence?}

Hybrid closed-loop systems have been studied for a number of years, expanding their role in type 1 diabetes as a means of achieving targeted glycaemic control whilst minimising the risk of hypoglycaemia [2]. The first commercially available closed-loop system was approved in 2016 by the US Food and Drug Administration (FDA) for patients aged 15 years and older [3]. A further landmark study established its safety and efficacy across the age spectrum to patients as young as 6 years [4]. However, evidence for hybrid closed-loop systems using open-source code is limited to observational studies, self-reported patient outcomes, and anecdotal reports with a conspicuous lack of robust clinical trials. Observational data emphasises the usefulness of this system in reducing hypoglycaemia, glycaemic variability, overnight controls and management burden with improvement in time-inrange (TIR), HbA1c [5, 6] and sleep [5, 7]. A Twitter analysis of posts from users and carers demonstrated improvement in HbA1c, glucose variability, diabetes burden and quality of life [7].

Further observational data from Korea [8] and Italy [9] showed reduction of time in hypoglycaemia and HbA1c. An analysis of CGM data of 80 OpenAPS users showed improvement in TIR, HbA1c, hypoglycaemia and glucose variability [10]. It has been estimated that users can save up to one day's time per month spent making decisions about diabetes management [11]. Table 2 summarises the pros and cons of DIY APS systems as compared to traditional sensor-augmented pump therapy.

It is, however, important to appreciate that most of the data is from highly motivated, techsavvy users and parents and thus subject to significant selection bias. There is no objective data from randomised clinical trials, on patients with variable degree of control.
In addition, there are no formal studies assessing risks of acute diabetic emergencies such as hypoglycaemia, hyperglycaemia and diabetic ketoacidosis (DKA), or long-term complications and mortality.

There is a growing community of DIY users with approximately 1896 individuals across the world looping with various combinations of hardware and software components [12], many of whom are very active on social media. The community works with a culture of "pay it forwards" whereby established users are encouraged to support new members and aims to be selfregulatory by discouraging unsafe practice [12]. Interoperability is the current mantra propagated by the looping community to device manufactures, encouraging them to develop devices that have the ability to talk to each other, using open communication protocols.

Tidepool, a non-profit organisation, is working to build an FDA-regulated version of Loop for iOS, to be available on the App store. They are working with commercially available pump manufactures in order to make Tidepool Loop compatible with various products.

\section{What Are the Ethical Concerns?}

There is no current framework that governs the accountability and responsibility on healthcare provision to DIY users. The technology has not been appraised or approved by regulatory bodies such as the National Institute of Clinical Excellence (NICE), FDA, and Medicine and Healthcare products Regulatory Agency (MHRA).

The Association of British Clinical Diabetologists (ABCD) encourages healthcare professionals (HCPs) to support their patient [13] but advises that the HCP should not recommend open source systems. The Juvenile Diabetes Research Foundation (JDRF), whilst recognising DIY technology as unregulated with potential safety concerns, respects current DIY users and appreciates that HCPs managing patients, who choose to be DIY users, will require specific training and enhanced indemnity to protect them from medicolegal liability [14]. Technology uptake is limited by HCP expertise which is 
dependent upon resources and protected time for learning and development [15]. Whilst current DIY APS users have high expertise in diabetes self-care, as numbers increase, HCPs may be asked to support individuals who lack a deeper understanding of the technology [16].

Many of the DIY components are available via the NHS, although some DIY users operate outof-warranty pumps, increasing the risk of system failure. HCPs need to advise that users should operate the DIY system at their own risk but also provide training in components provided by the NHS [14]. HCPs are duty-bound to inform the patients/carers about the intended use of medical products and the risk of harm from the use of products in an unregulated, non-conventional manner. There have been concerns about the accuracy of the FreeStyle Libre device for looping, particularly if safe calibration does not occur [17].

Around a third of DIY APS users are children using systems built by parents or family members [12]. HCPs working with children and young people with diabetes will need to consider safeguarding issues when discussing the risk and benefits of DIY systems with patients and families. However, for most families, using this technology will be a decision taken in the best interests of their child after extensive research and consideration.

\section{CONCLUSION}

The use of DIY hybrid closed-loops systems continues to grow in popularity with patients and their families. It is vital that HCPs have some understanding of these systems and are able to discuss possible risks and benefits with their patients and support them where this is feasible.

\section{ACKNOWLEDGEMENTS}

The authors thank all the participants for their contribution to this article.
Funding. No funding or sponsorship was received for the write up or publication of this article.

Authorship. All named authors meet the International Committee of Medical Journal Editors (ICMJE) criteria for authorship for this article, take responsibility for the integrity of the work as a whole, and have given their approval for this version to be published.

Disclosures. The physician authors (S. Haris Ahmed, David L. Ewins, Jane Bridges and Alison Timmis) as well as the patients (Ms. Nicola Payne, Mr. Cormac Mooney and Ms. Claire MacGregor, patient Harry Mullins' mum) have nothing to disclose.

Compliance with Ethics Guidelines. This article is based on previously conducted studies and does not contain any studies with human participants or animals performed by any of the authors.

Data Availability. Data sharing is not applicable to this article as no datasets were generated or analysed during the current study.

Open Access. This article is licensed under a Creative Commons Attribution-NonCommercial 4.0 International License, which permits any non-commercial use, sharing, adaptation, distribution and reproduction in any medium or format, as long as you give appropriate credit to the original author(s) and the source, provide a link to the Creative Commons licence, and indicate if changes were made. The images or other third party material in this article are included in the article's Creative Commons licence, unless indicated otherwise in a credit line to the material. If material is not included in the article's Creative Commons licence and your intended use is not permitted by statutory regulation or exceeds the permitted use, you will need to obtain permission directly from the copyright holder. To view a copy of this licence, visit http://creativecommons.org/licenses/by$\mathrm{nc} / 4.0 /$. 


\section{REFERENCES}

1. Lewis D. History and perspective on DIY closed looping. J Diabetes Sci Technol. 2019;13(4):790-3.

2. Bekiari E, Kitsios K, Thabit H, et al. Artificial pancreas treatment for outpatients with type 1 diabetes: systematic review and meta-analysis. BMJ. 2018;361:k1310. http://www.bmj.com/content/ 361/bmj.k1310.abstract.

3. Bergenstal RM, Garg S, Weinzimer SA, et al. Safety of a hybrid closed-loop insulin delivery system in patients with type 1 diabetes. JAMA. 2016;316(13): 1407-8.

4. Tauschmann M, Thabit H, Bally L, et al. Closedloop insulin delivery in suboptimally controlled type 1 diabetes: a multicentre, 12-week randomised trial. Lancet. 2018;392(10155):1321-9.

5. Lewis D, Leibrand S. Real-world use of open source artificial pancreas systems. J Diabetes Sci Technol. 2016;10:1411.

6. Lewis DM, Swain RS, Donner TW. Improvements in A1C and time-in-range in DIY Closed-Loop (OpenAPS) Users. Diabetes. 2018;67(Supplement 1):352OR. http://diabetes.diabetesjournals.org/content/ 67/Supplement_1/352-OR.abstract.

7. Litchman ML, Lewis D, Kelly LA, Gee PM. Twitter Analysis of \#OpenAPS DIY artificial pancreas technology use suggests improved A1C and quality of life. J Diabetes Sci Technol. 2019;13(2):164-70.

8. Choi SB, Hong ES, Noh YH. Open artificial pancreas system reduced hypoglycemia and improved glycemic control in patients with type 1 diabetes. Diabetes. 2018;67(Supplement 1):964-P. http:// diabetes.diabetesjournals.org/content/67/Supplem ent_1/964-P.abstract.

9. Provenzano V, Guastamacchia E, Brancato D, et al. Closing the loop with OpenAPS in people with type 1 diabetes-experience from Italy. Diabetes.
2018;67(Supplement 1):993-P. http://diabetes. diabetesjournals.org/content/67/Supplement_1/ 993-P.abstract.

10. Melmer A, Züger T, Lewis DM, Leibrand SM, Laimer M. 76-OR: In-depth review of glycemic control and glycemic variability in people with type 1 diabetes using open source artificial pancreas systems. Diabetes. 2019;68(Supplement 1):76-OR. http:// diabetes.diabetesjournals.org/content/68/Supplem ent_1/76-OR.abstract.

11. Street T. \#WeAreNotWaiting at the Type1AndTech Conference. http://circles-of-blue.winchcombe.org/ index.php/2018/11/11/wearenotwaiting-at-thetype1andtech-conference/. Accessed June 6, 2020.

12. OPENAPS.ORG. https://openaps.org/outcomes/. Accessed June 6, 2020.

13. Association of British Clinical Diabetologists. Do-ityourself artificial pancreas system (DIY APS) audit. Diabetol. 2020. https://abcd.care/DIYAPS. Accessed June 6, 2020.

14. Juvenile Diabetes Research Foundation(JDRF). JDRF UK position statement on type 1 diabetes DIY technologies. https://jdrf.org.uk/about-us/positionstatements-reports/position-statements/jdrfs-uk-po sition-statement-on-type-1-diabetes-diy-technolog ies/. Accessed June 6, 2020.

15. James S, Perry L, Gallagher R, Lowe J. Diabetes educators' intended and reported use of common diabetes-related technologies: discrepancies and dissonance. J Diabetes Sci Technol. 2016;10(6): 1277-86. 1932296816646798. https://doi.org/10.1177/

16. Wilmot EG, Danne T. DIY artificial pancreas systems: the clinician perspective. Lancet Diabetes Endocrinol. 2020;8(3):183-5.

17. Street T. Diabetech-diabetes and technology. 2019. https://www.diabettech.com/xdrip/libre-accu racy-and-calibration-using-a-third-party-transmitte r-a-case-study. Accessed June 6, 2020. 